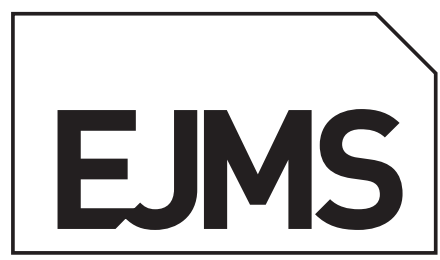

\title{
Composition, sequencing and ion mobility mass spectrometry of heparan sulfate- like octasaccharide isomers differing in glucuronic and iduronic acid content
}

\author{
Rebecca L. Miller, ${ }^{a, b}$ Wei Wei, ${ }^{a}$ Ralf Schwörer, ${ }^{c}$ Olga V. Zubkova, ${ }^{c}$ Peter C. Tyler, ${ }^{c}$ Jeremy E. Turnbull ${ }^{b}$ and \\ Julie A. Leary ${ }^{\mathrm{a}}$ \\ aDepartments of Molecular and Cellular Biology and Chemistry, University of California, 1 Shields Dr. Davis, CA 95616 USA.
E-mail: jaleary@ucdavis.edu \\ ${ }^{b}$ Current address: Centre for Glycobiology, Institute of Integrative Biology, University of Liverpool, Crown Street, Liverpool L69 7ZB, UK. \\ E-mail: becky@liv.ac.uk
}

${ }^{\mathrm{c}}$ Ferrier Research Institute, Victoria University of Wellington, 69 Gracefield Road, Gracefield, Lower Hutt 5010, New Zealand

\begin{abstract}
Here we report ion mobility mass spectrometry (IMMS) separation and tandem mass spectrometry (MS ${ }^{2}$ ) sequencing methods used to analyze and differentiate six synthetically produced heparin/heparan sulfate (HS)-like octasaccharide (dp8) isomeric structures. These structures are isomeric with regard to either glucuronic acid (GlcA) or iduronic acid (IdoA) residues at various positions. IMMS analysis showed that a fully GlcA structure exhibited a more compact conformation, whereas the fully IdoA structure was more extended. Interestingly, the change from IdoA to GlcA in specific locations resulted in strong conformational distortions. $\mathrm{MS}^{2}$ of the six isomers showed very different spectra with unique sets of diagnostic product ions. Analysis of $\mathrm{MS}^{2}$ product ion spectra suggests that the GlcA group correlated with the formation of a glycosidic product ion under lower energy conditions. This resulted in an earlier product ion formation and more intense product ions. Importantly, this knowledge enabled a complete sequencing of the positions of GlcA and IdoA in each of the four positions located in each unique dp8 structure.
\end{abstract}

Keywords: glycosaminoglycan, heparan sulfate, IMMS, MS², electron detachment dissociation, arrival time distribution, glucuronic acid, iduronic acid

\section{Introduction}

Structural characterization of heparin and heparan sulfate (HS) and their various binding partners is of significant interest to the glycobiology community. Heparin and HS are highly interactive molecules and regulators of biological systems, present in injury, ${ }^{1,2}$ anticoagulation, ${ }^{3}$ defense, ${ }^{4,5}$ angiogenesis, ${ }^{6,7}$ growth and development, ${ }^{8}$ and tumor metastasis. ${ }^{9}$ As a consequence of this, there is a growing imperative to understand their structure-function relationships, and thus the need for methods of sequencing these complex glycans. ${ }^{10,11}$ Heparin and HS are highly anionic molecules that are part of the glycosaminoglycan family; they differ not only in their levels and positions of sulfation, but also in their glucuronic acid (GlcA) and iduronic acid (IdoA) content and location. ${ }^{12,13}$ Both contain variable levels of sulfation on the glucosamine residues at carbons 2 and 6 (and rarely 3), while the $\mathrm{N}$-positions of glucosamines can be modified with either a sulfate or an 
acetate (and sometimes a free amino group). Heparin tends to be a more heavily sulfated molecule laverage of $\sim 2.6$ sulfates per disaccharide) with L-IdoA predominating over D-GlcA ( 9: 1), whereas HS is more heterogeneous with fewer negative charges per disaccharide (typically 1-2 sulfates per disaccharide unit) and with D-GlcA predominating. These key differences have been related to the protein interactions and the regulatory functions of heparin and HS. ${ }^{14-18}$

Currently, only a handful of heparin and HS oligosaccharide structures have been isolated and their sulfation patterns determined through methods such as compositional analysis, ${ }^{19-26}$ enzymatic methods, ${ }^{27}$ tandem mass spectrometry $\left(\mathrm{MS}^{2}\right)^{23,28}$ and nuclear magnetic resonance (NMR) spectrometry. ${ }^{29}$ There has been much less success in the determination of IdoA and GlcA positions, although NMR spectrometry has been used to determine GlcA and IdoA positions in limited studies on small saccharides (dp4). ${ }^{30-33}$ In combination with NMR validation, MS analysis is progressing with techniques such as negative electron-transfer dissociation (NETD),34,35 electron detachment dissociation (EDD), 33,36,37 electron induced dissociation (EID) ${ }^{38}$ and infrared multiphoton dissociation (IRMPD). ${ }^{36}$ Collisionally acticated dissociation (CAD) as shown some degree of success in determining GlcA/ IdoA positions in dp4 and dp6 saccharides. ${ }^{24,33}$ These techniques confirmed glycosidic bond cleavages via $B, C, Y$, and $Z$ ions, and $\mathrm{CO}_{2}$ losses, with the GlcA structures showing more dissociation ions.

IdoA acid tends to be located in the more highly sulfated regions containing $\mathrm{N}$-sulfated glucosamines, whereas GlcA is generally found in less sulfated regions with GlcNAc residues. ${ }^{39}$ In principle, both of these monosaccharides can adopt several different boat and chair conformations. However, the chair form is generally more favored, because of less crowding and steric hindrance. In the chair conformation, the functional group can be in either the equatorial position, which projects out from the ring, or in the axial position, which projects either upward or downward from the ring. Uronic acids in $\mathrm{HS}$ have three different conformations: the ${ }^{1} \mathrm{C}_{4}$ and ${ }^{4} \mathrm{C}_{1}$ chairs and a ${ }^{2} \mathrm{~S}_{0}$ skew boat, with ${ }^{4} \mathrm{C}_{1}$ being the most common. ${ }^{40,41}$ NMR studies of heparin and HS have shown that GlcA tends to adopt a more rigid ring with a preference for the ${ }^{4} C_{1}$ chair conformation, whereas the iduronate residue tends to be more flexible and is able to adopt a mixture of both ${ }^{1} \mathrm{C}_{4}$ and ${ }^{4} \mathrm{C}_{1}$ chair conformations, as well as the ${ }^{2} \mathrm{~S}_{0}$ skew boat conformation. ${ }^{40,41}$ The conformational equilibrium of the monosaccharide ring between these three forms is very dependent on sulfation of the C-2 oxygen and the neighboring glucosamine. ${ }^{31}$ The glycosidic bond is relatively rigid in oligomers with $\mathrm{N}$-sulfated glucosamines compared to those with $\mathrm{N}$-acetyl groups, because of the formation of a transient hydrogen bond between the oxygen of the $\mathrm{N}$-sulfate and the $\mathrm{C}-3$ hydroxyl hydrogen of the neighboring uronic acids. ${ }^{31,42}$ Overall, HS is able to adopt a variety of conformations in solution, a property that is thought to be important for biological interactions.

Although ion mobility mass spectrometry (IMMS) has been used rather frequently in the analysis of proteins and, more recently, protein-carbohydrate complexes, ${ }^{25,43-47}$ it is not commonly used for the analysis of GAG. Herein, we have examined the potential of IMMS to separate and correctly identify octasaccharide (dp8) HS structural isomers with differing positions of the GlCA and IdoA residues. The six octasaccharides discussed herein were produced utilizing a recently described chemical synthesis strategy. ${ }^{48}$ In addition, we used $\mathrm{MS}^{2}$ to sequence unambiguously the positions of GlcA and IdoA, which previously has been intractable in compounds larger than tetrasaccharides.

\section{Experimental Reagents}

High-performance liquid chromatography grade water, acetonitrile, and ammonium hydroxide were used and purchased from Fisher (Santa Clara, CA).

\section{IMMS of six isomeric octasaccharides}

IMMS was performed on either a Synapt G1 or G2 mass spectrometer equipped with a T-wave mobility cell (Waters Corp., Milford, MA). The octasaccharide concentration of compounds 1 to 6 (Figure 1) was $5 \mu \mathrm{M}$ in water/acetonitrile (50/50 v/v) with $500 \mathrm{mM}$ ammonium hydroxide. The sample was loaded into borosilicate electrospray tips made in house. Briefly, the borosilicate tubes (no. 1B120F-4) were purchased from World Precision Instruments, Inc. (Sarasota, FL), and were pulled using a Sutter P97 Flaming/Brown micropipette puller (Ringmer, Sussex, UK). The tips were gold coated using a Quorum Technologies SC7640 sputter coater (Ringmer, Sussex, UKJ. The mass spectra were acquired in the negative ion mode using a capillary voltage at $0.55 \mathrm{kV}$, a sample cone voltage at $7 \mathrm{~V}$, and an extraction cone voltage at $0.6 \mathrm{~V}$. The ion mobility parameters were optimized for the octasaccharide separation and all the parameters are given in the Supplementary Materials. The collisional cross-section data were acquired and confirmed using the Synapt G2 with optimized ion mobility conditions (Supplementary Materials). MS² was performed on selected ions and collisionally activated at $22 \mathrm{~V}$ in the transfer cell with the mobility cell turned off in order to produce comparable collision-induced dissociation (CID) data for each isomer.

\section{Ion mobility calibration curve}

Measured drift times were converted to collisional crosssection values through a calibration curve of oligonucleotide standards T7, C7, and $(\mathrm{AT})_{3}$. Accurate IMMS collisional crosssections of $T 7, C 7$, and $(A T)_{3}$ were previously calculated by the Michael T. Bowers Laboratory at the University of California, Santa Barbara. The standards T7, C7, and $(\mathrm{AT})_{3}$ were mixed at a concentration of $20 \mu \mathrm{M}$ in $10 \mathrm{mM} \mathrm{NH}_{4} \mathrm{OH}$ in methanol:water (1:1) and were subjected to identical IMMS experimental parameters as compounds $1-6$. The arrival time distributions (ATDs) from T7, C7, and $(A T)_{3}$ were plotted, and a linear equation was deduced, $y=145.66 x+480.6$, with $R^{2}=0.99$, where 


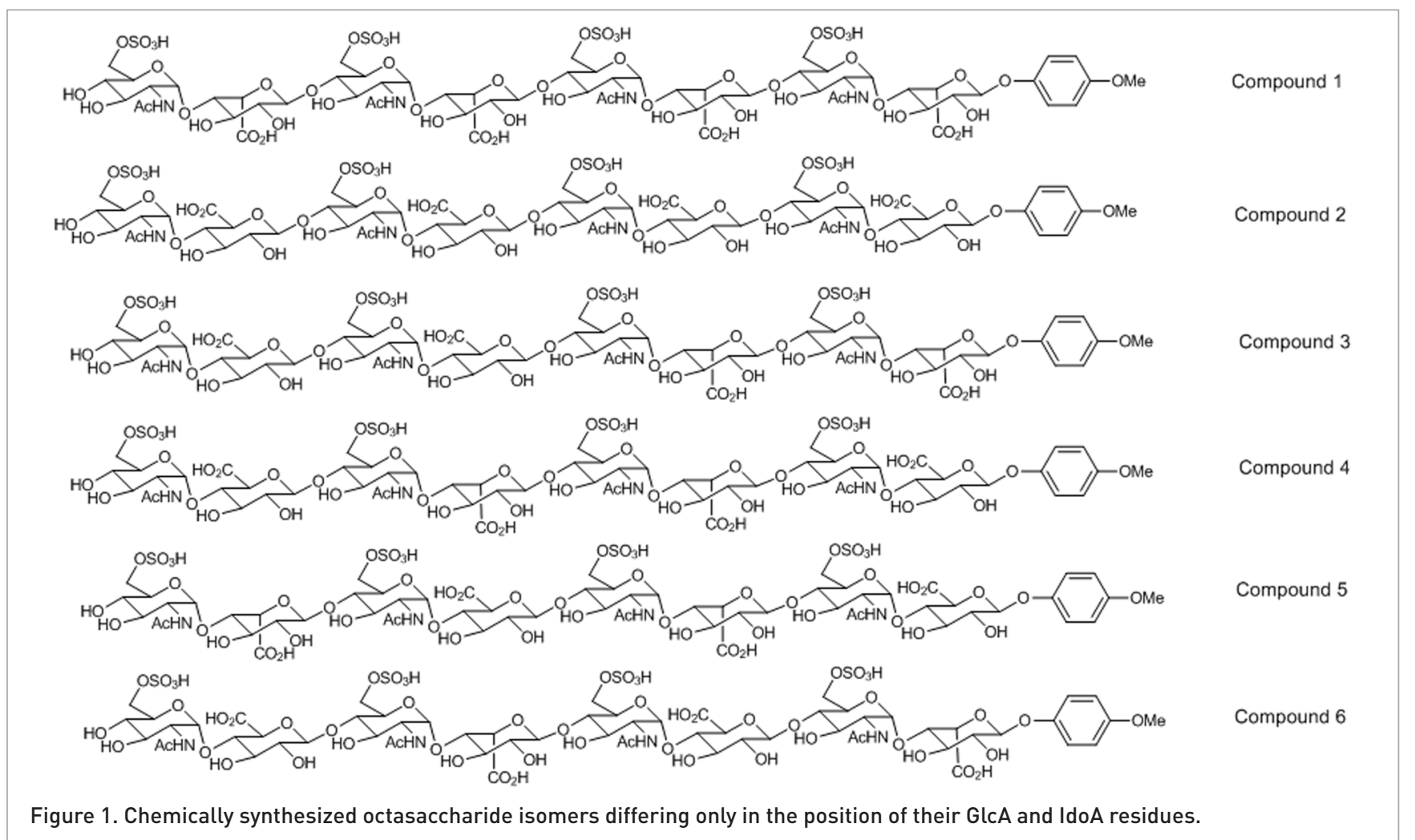

$y$ represents the corrected collisional cross-sectional area (CCS) of each oligonucleotide and $x$ represents the corrected ATD of each oligonucleotide. Compounds 1-6 were subsequently assessed from this linear equation.

\section{Results and discussion IMMS of synthetic dp8 saccharides}

IMMS was initially used to characterize six fully synthetic octasaccharides (Figure 1) containing 6-O-sulfated $\mathrm{N}$-acetylglucosamine residues, which were synthesized with a methoxyphenol tag at the reducing end. As can be seen, the differences among these structures is solely the positioning of the IdoA or GlcA residues; thus, the only structural differences are in the axial or equatorial positions of the acidic functional group. The IMMS procedure was first developed and tested with the two simplest compounds containing either all IdoA residues (compound 1, I-I-I-I-Tag) or all GlcA residues (compound 2, G-G-G-G-Tag), in an effort to separate and unambiguously characterize these stereoisomers. If the conformation of a fully IdoA-containing dp8 (compound 1) and a fully GlcA-containing dp8 (compound 2) are sufficiently different, IMMS should permit some degree of separation between the two compounds. We show herein that it is possible to isolate and separate these two compounds as evidenced by the difference in ATDs of the $3^{-}$charge state of each individual octasaccharide, both separately and in a mixture [Figure 2(a) and (b)]. This difference in ATD is somewhat expected as NMR studies of heparin and HS have shown that GlcA tends to be more rigid with a preference for the ${ }^{4} C_{1}$ chair conformation, whereas the IdoA residue is more flexible. The more flexible IdoA is able to adopt a mixture of ${ }^{1} \mathrm{C}_{4},{ }^{4} \mathrm{C}_{1}$, and ${ }^{2} \mathrm{~S}_{0}$ conformations.

The IdoA-containing octasaccharide appears to adopt a conformation that is likely more extended than the GlcA structure, and results in an ATD of $4.33 \mathrm{~ms}\left(\mathrm{CCS}=342 \AA^{2}\right.$ ) (Figure 2). Adjustment from the axial to the equatorial position of the carboxylate leads to a rather significant $0.81 \mathrm{~ms}$ (or $29 \AA^{2}$ difference in CCS) separation between the two isomers, with the GlcA isomer having a more compact conformation [ATD of $\left.3.52 \mathrm{~ms}\left(313 \AA^{2}\right)\right]$. The experiment was repeated three times on different days and showed good reproducibility [Supplementary Figure $1(a)$ and $1(b)$ ].

\section{$\mathrm{MS}^{2}$ of two dp8 isomers, compounds 1 and 2}

Having established the appropriate parameters for stereoisomeric separation, we then proceeded to determine the best conditions for $\mathrm{MS}^{2}$ differentiation. Figure 3(a) and (b) show the $M^{2}$ spectra for compound 1 and compound 2, respectively. The precursor ion at $\mathrm{m} / \mathrm{z} 652.44,[\mathrm{M}-3 \mathrm{H}]^{3-}$ for each isomer was isolated and collisionally activated in the transfer cell (with the mobility cell turned off). Interestingly, the structure that contained only GlcA showed a glycosidic bond dissociation at $12 \mathrm{~V}$, whereas the structure containing IdoA did not dissociate until subjected to $18 \mathrm{~V}$. Increasing the collision energy to $22 \mathrm{~V}$ resulted in substantially more dissociation (dissociation ions were assigned using the Domon and Costello nomenclature ${ }^{49}$ ). The $\mathrm{MS}^{2}$ data at $22 \mathrm{~V}$ showed remarkably different spectra for the two structures; different diagnostic product 

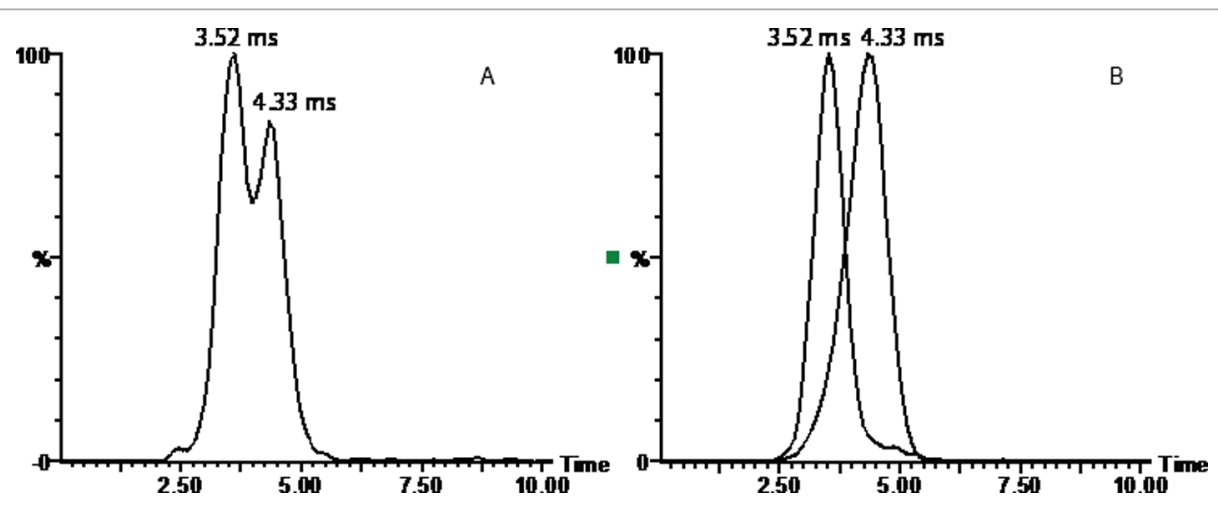

Figure 2. Ion mobility separation of dp8 isomers containing exclusively either GlcA or IdoA. (a) ATD for the mixture of compound 1 (I-I-I-I-Tag) and compound 2 (G-G-G-G-Tag) at the $3^{-}$charge state. Compound 1 appears to displays an extended conformation resulting in an ATD of $4.33 \mathrm{~ms}$, whereas compound 2 displays a compact conformation resulting in a shorter ATD of $3.52 \mathrm{~ms}$. (b) ATD of the $3^{-}$ charge states of compound 1 and compound 2 run separately and then overlaid. The two dp8 isomers show a separation of $0.81 \mathrm{~ms}$ with a CCS difference of $29 \AA^{2}$.

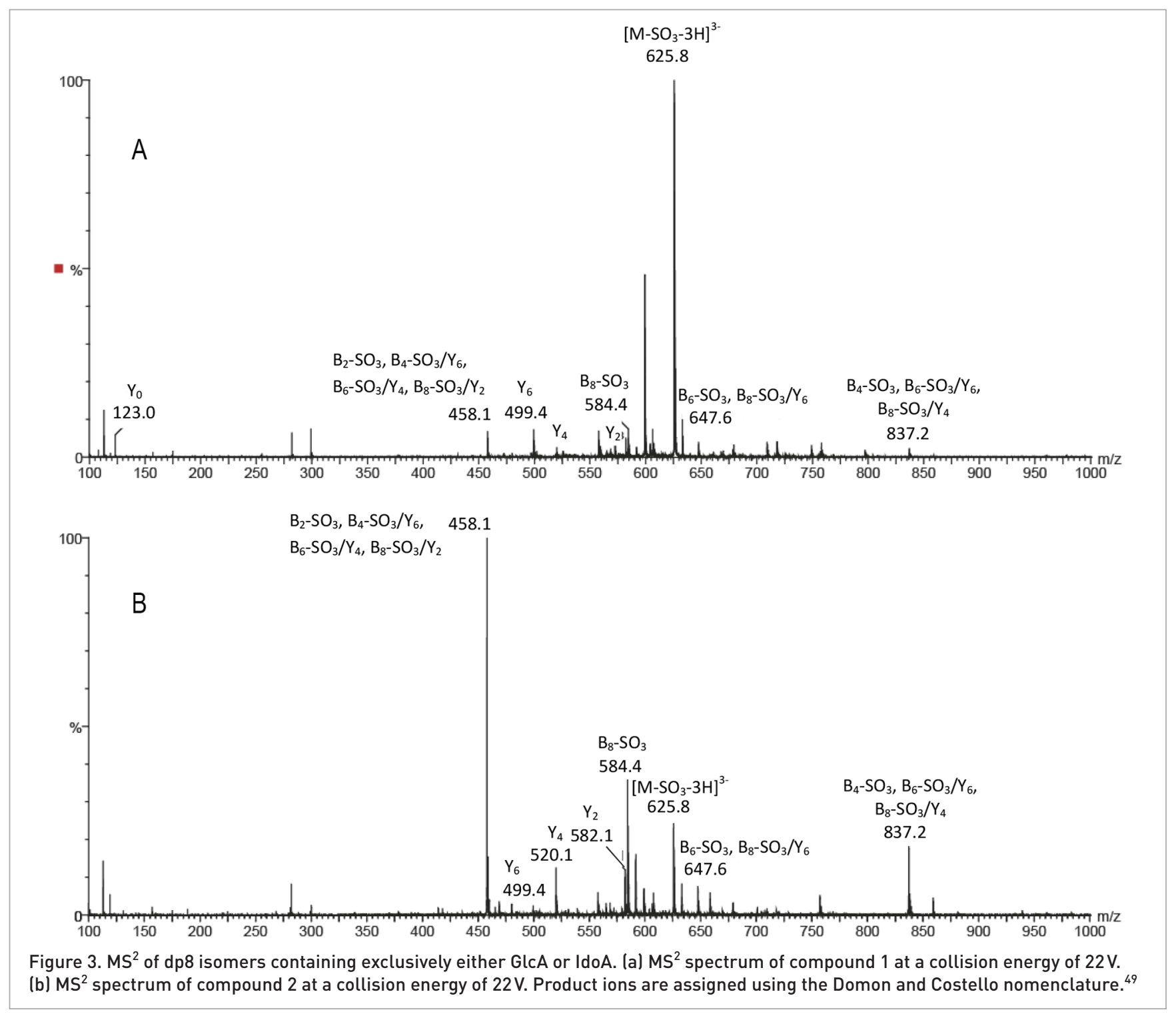


ions were produced and the intensities of the common product ions were also quite different [Figure 3(a) and (b)].

In sequencing repeating structural moieties, it is very difficult to define precisely each ion, as there can be several possible structures for each $\mathrm{m} / \mathrm{z}$ value. Techniques such as NETD, ${ }^{34,35}$ EDD, $^{33,36,37}$ EID, $^{38}$ IRMPD, $^{36}$ and CAD, ${ }^{24,33}$ used to dissociate GlcA- and IdoA-containing structures, resulted in similar problems. However, the presence of the reducing end tag facilitates differentiation of each structure with the resulting ions specific to each isomer.

In both compounds, $Y$ and $Z$ ions are distinguished by the presence of the tag. Intact $Y$ ions have a unique $\mathrm{m} / \mathrm{z}$ ratio, and various $B$ and $B / Y$ fragments can produce ions at $m / z$ 458.1. As neither $Z$ nor $C$ ion fragments were identified, the number of structures with the same $\mathrm{m} / \mathrm{z}$ of 458.1 is reduced. Diagnostic ions with a single sulfate loss were selected to distinguish between compounds 1 and 2: eight ring $\left(\mathrm{B}_{8}-\mathrm{SO}_{3}{ }^{3-}, \mathrm{m} / \mathrm{z}\right.$ 584.4), six-ring $\left(\mathrm{B}_{6}-\mathrm{SO}_{3}, \mathrm{~B}_{8}-\mathrm{SO}_{3} / \mathrm{Y}_{6}{ }^{2-}, \mathrm{m} / z\right.$ 647.6) and the four-ring $\left(\mathrm{B}_{4}-\mathrm{SO}_{3}, \mathrm{~B}_{6}-\mathrm{SO}_{3} / \mathrm{Y}_{6}, \mathrm{~B}_{8}-\mathrm{SO}_{3} / \mathrm{Y}_{4}{ }^{1-}, \mathrm{m} / \mathrm{z}\right.$ 837.2).

For both compounds 1 and 2, the intensities of the glycosidic bond cleavages identified were represented as a ratio of the glycosidic bond product to the precursor ion. For example, the intensity of $\mathrm{m} / \mathrm{z} 123.0\left(\mathrm{Y}_{0}^{-}\right)$was represented as a ratio of the intensity of the precursor ion, $\mathrm{m} / \mathrm{z} 625.8\left[\mathrm{M}--\mathrm{SO}_{3}-3 \mathrm{H}\right]^{3-}$. After each product ion ratio was determined, compound 2 was allocated a value of $100 \%$ and the other glycosidic bond cleavages were represented as a relative percentage. This normalization was implemented for all the glycosidic bond cleavages identified, and is shown in Figure 4. When comparing compounds 1 and $2, \mathrm{~B}$ fragments $\left(\mathrm{B}_{2}-\mathrm{SO}_{3}, \mathrm{~B}_{4}-\mathrm{SO}_{3}, \mathrm{~B}_{6}-\mathrm{SO}_{3}, \mathrm{~B}_{8}-\mathrm{SO}_{3}\right), \mathrm{B} / \mathrm{Y}$ fragments $\left(\mathrm{B}_{2}-\mathrm{SO}_{3}, \mathrm{~B}_{4}-\mathrm{SO}_{3}, \mathrm{~B}_{6}-\mathrm{SO}_{3}, \mathrm{~B}_{8}-\mathrm{SO}_{3} / \mathrm{Y}_{6,4,2,0}\right.$ combinations) and $Y$ fragments were low in compound 1 relative to those in compound 2 (Figure 3 and Figure 4). When comparing $\mathrm{m} / \mathrm{z} 123.0\left(\mathrm{Y}_{0}^{-}\right)$and its complementary ion $\mathrm{m} / \mathrm{z} 584.4\left(\mathrm{~B}_{8}-\mathrm{SO}_{3}{ }^{3-}\right)$, it was interesting to observe that compound 1 had a higher intensity of $\mathrm{Y}_{0}^{-}$and a lower intensity of $\mathrm{B}_{8}-\mathrm{SO}_{3}{ }^{3-}$ compared to compound 2 (Figure 4). The observation that the intensities of $\mathrm{B}$ fragments $\left(\mathrm{B}_{2}-\mathrm{SO}_{3}, \mathrm{~B}_{4}-\mathrm{SO}_{3}, \mathrm{~B}_{6}-\mathrm{SO}_{3}, \mathrm{~B}_{8}-\mathrm{SO}_{3}\right)$ and $\mathrm{B} / \mathrm{Y}$ fragments $\left(\mathrm{B}_{2}-\mathrm{SO}_{3}, \mathrm{~B}_{4}-\mathrm{SO}_{3}, \mathrm{~B}_{6}-\mathrm{SO}_{3}, \mathrm{~B}_{8}-\mathrm{SO}_{3} / \mathrm{Y}_{6,4,2,0}\right.$ combinations) were always higher in compound 2 (Figure 4) suggests that a glycosidic bond-cleavage mechanism near the GlcA produced product ions with a neutral tag, causing limited detection of $Y_{0}^{-}$. The other $B$ and $B / Y$ ions appear unaffected. The detection of $Y_{0}^{-}$in compound 1 , the IdoA-containing isomer, suggests that the tag retains the $\mathrm{O}^{-}$after glycosidic bond cleavage.

Based on the dramatic differences in the intensity of $B$ and $B / Y$ fragments, and especially $Y_{0}^{-}$ions, these data clearly indicate that the product ions produced via the CID of compounds 1 and 2 were formed through different mechanisms. As both compounds showed no $\mathrm{CO}_{2}$ loss, which is the route observed when fragmentation occurs through NETD, ${ }^{34,35}$ EDD, ${ }^{33,36,37}$ EID, ${ }^{38}$ and IRMPD, ${ }^{36}$ we propose that the formation of the $B$ ions during the CID of GlcA likely results in the formation of the epoxide at $\mathrm{C}-2$ with concomitant glycosidic cleavage. It is likely that this process is much more favorable for the equatorial substituent and less so for the carboxylate in the axial position. Further mechanistic insights require $0^{18}$ and deuterium labeling experiments, which will be the focus of subsequent studies.

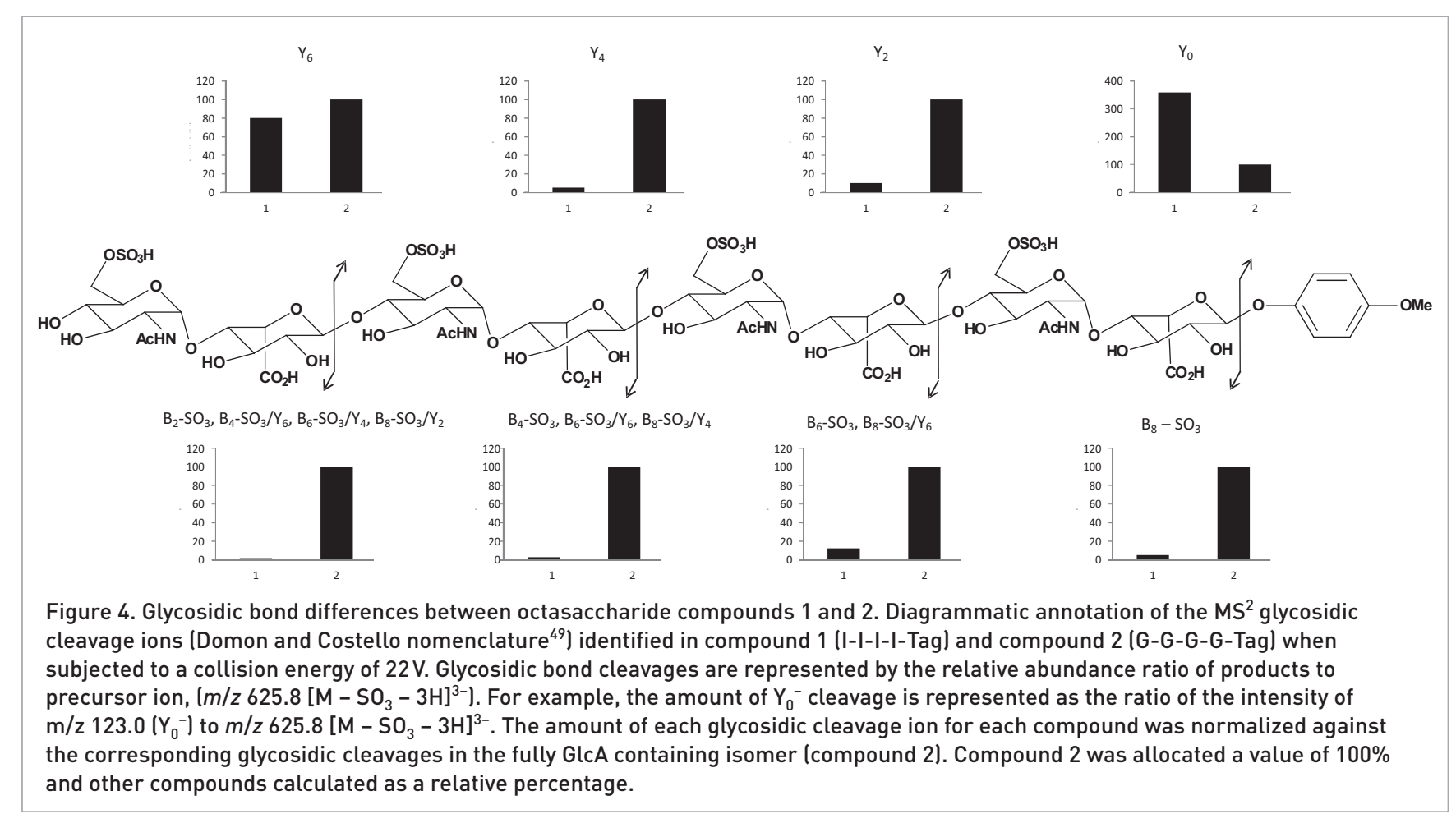




\section{Ion mobility of a library of six dp8 isomers with divergent positions of GlcA/IdoA residues}

Ion mobility of the six different GlcA and IdoA isomers showed different ATDs (Figure 5). Compounds 1 and 2 are discussed above. Compound 3, G-G-I-I-Tag, and Compound 4, G-I-IG-Tag, showed ATDs of $3.47 \mathrm{~ms}\left(310 \AA^{2}\right)$ and $3.73 \mathrm{~ms}\left(319 \AA^{2}\right)$, respectively [Figure $5(\mathrm{c})$ and (d)], with a difference of only $0.26 \mathrm{~ms}\left(9 \AA^{2}\right)$, which is relatively small in comparison to compounds 1 and 2. Compound 5, I-G-I-G-Tag, and compound 6, G-I-G-I-Tag, are particularly interesting as they produced somewhat unexpected ATDs of $3.41 \mathrm{~ms}\left(308 \AA^{2}\right)$ and $4.33 \mathrm{~ms}$ $\left(342 \AA^{2}\right.$ ), respectively [Figure $5(\mathrm{e})$ and $\left.(\mathrm{f})\right]$. The ion mobility separation of these latter two compounds was $0.92 \mathrm{~ms}\left(34 \AA^{2}\right)$. greater than the separation of compounds 1 and 2. The arrival times of the fully IdoA and fully GlcA structures suggests that the fully IdoA structure has a more extended conformation, whereas the fully GlcA structure has a more compact conformation. Of note is that each of the isomers that contained a reducing end GlcA-tag had very similar arrival times, and all showed a more compact conformation than those isomers with a reducing end IdoA-tag. The exception to this is compound 3. We believe that the short ATD for this isomer may result from the compact and likely constrained conformations of both GlcA rings on the non-reducing ring, which is likely to cause a shortening around the partial 1-4 linked helix that is formed with the G-G-I-I sequence.

The ion mobility separation of these six isomers highlights a significant challenge in the glycobiology field, i.e. corre-

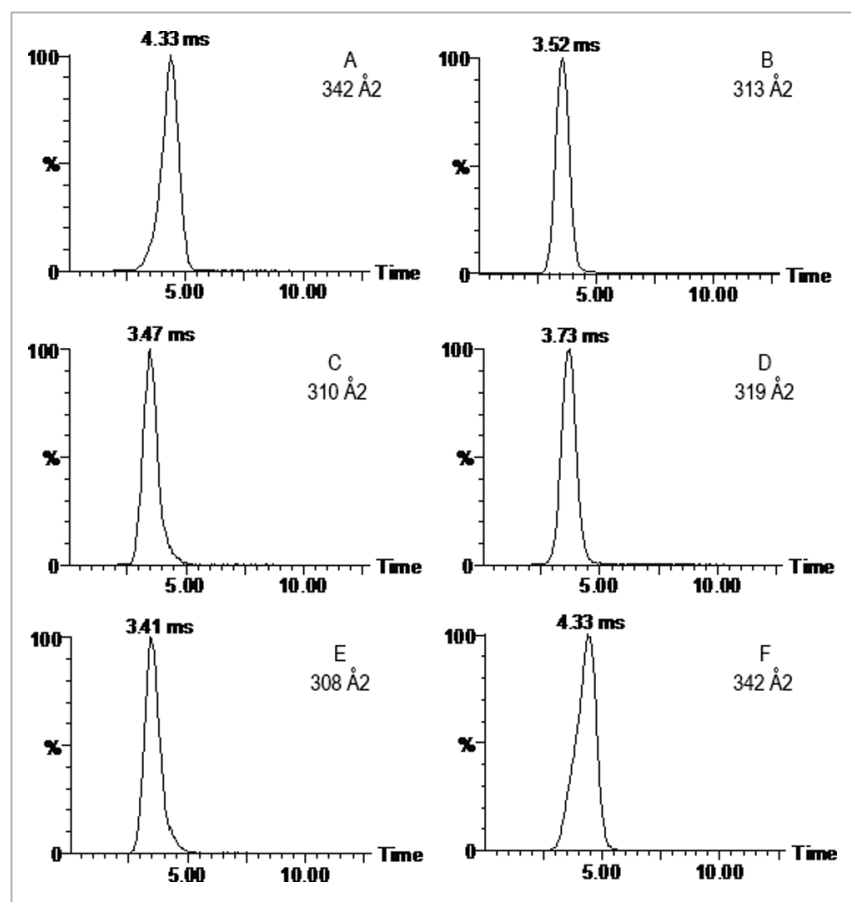

Figure 5. Ion mobility separation of six dp8 isomers containing positional differences in GlcA and IdoA. (a)-(f) ATD and calculated collisional cross-section for compounds 1-6, respectively. lating sequence with conformation. It is well-known that both sequence and conformation are essential to biological interaction, activity, and functional outcomes. Nevertheless, techniques to examine these correlations are limited. We have demonstrated that ion mobility of three different sequences, compounds 2, 4, and 5, can occupy a similar hydrodynamic volume when the tagged reducing end ring possesses the same conformation. The ion mobility of two structures, I-G-IG-Tag and G-I-G-I-Tag, that might be expected to have similar, if not the same, hydrodynamic volumes revealed very distinct differences, again supporting the fact that the tag on the reducing ring helps to anchor the conformation. These initial data provide an initial step toward the understanding of biological interactions via examining correlations between structural conformation and sequence.

\section{$M S^{2}$ of dp8 saccharides with divergent positions of GlcA/IdoA residues}

The $\mathrm{MS}^{2}$ of compounds 3-6 (Supplementary Figure 2) were analyzed and evaluated based on the sequencing knowledge determined with compounds 1 and 2. A glycosidic cleavage event occurs at a lower energy when a GlcA is adjacent, and thus glycosidic cleavages will occur at a higher frequency when a GlcA is present compared to when an IdoA is. This knowledge provides the background to predict the $\mathrm{MS}^{2}$ outcomes for other GlcA- and IdoA-containing isomers. For example, |-|-|-|-Tag would have no low energy state cleavage events, as GlcA is not present, whereas I-I-I-G-Tag would have a high intensity of $\mathrm{B}_{8}{ }^{3-}$ and a low intensity of $\mathrm{Y}_{0}{ }^{-}$as a cleavage event would occur on the glycosidic bond to the right of GlcA. If the sequence were G-G-I-G-Tag, the $\mathrm{B}_{8}-\mathrm{SO}_{3}{ }^{3-}$ ion would be formed. However, $\mathrm{B}_{8}-\mathrm{SO}_{3}{ }^{3-}$ would be further cleaved, producing $\mathrm{B}_{8}-\mathrm{SO}_{3} / \mathrm{Y}_{4}^{-}(\mathrm{I}-\mathrm{G})$ and $\mathrm{B}_{4}-\mathrm{SO}_{3}^{-}(\mathrm{G}-\mathrm{G})$, among which $\mathrm{B}_{4}-\mathrm{SO}_{3}^{-}(\mathrm{G}-\mathrm{G})$ would be cleaved into $\mathrm{B}_{4}-\mathrm{SO}_{3} / \mathrm{Y}_{6}^{-}(\mathrm{G})$ and $\mathrm{B}_{2}-\mathrm{SO}_{3}{ }^{-}(\mathrm{G})$. The intensity of $\mathrm{B}, \mathrm{B} / \mathrm{Y}$, and $Y$ fragment ions should produce an unambiguous characterization of a particular GlcA- and IdoA-containing sequence. The in silico expectations for product ions based on our rationale are summarized in Table 1.

The intensity distribution of $B, B / Y$, and $Y$ fragment ions detected for each dp8 compound should enable sequencing, even though our understanding of how conformation affects sequencing is still limited. Glycosidic bond cleavages are represented by the relative abundance ratio of products to precursor ion, $\left(\mathrm{m} / \mathrm{z} 625.8\left[\mathrm{M}-\mathrm{SO}_{3}-3 \mathrm{H}\right]^{3-}\right)$. For example, the amount of $\mathrm{Y}_{0}^{-}$cleavage is represented as the ratio of the intensity of $\mathrm{m} / \mathrm{z} 123.0\left(\mathrm{Y}_{0}^{-}\right)$to $\mathrm{m} / \mathrm{z} 625.8\left[\mathrm{M}-\mathrm{SO}_{3}-3 \mathrm{H}\right]^{3-}$. The amount of each glycosidic cleavage ion for each compound was normalized against the corresponding glycosidic cleavages in the fully GlcA-containing isomer (compound 2). The fully IdoA structure (compound 1) was used as a control for the amount of glycosidic ions formed through the IdoA mechanism. The comparison of each glycosidic ion from compounds 3 to 6 with the fully IdoA structure and with the fully GlcA structure should permit whether the fragment ion was formed through a GlcA cleavage mechanism or through 
Table 1. Theoretical dissociation pattern for all possible dp8 structures that contain either a GlcA or an IdoA at each uronic acid position. Dissociation patterns of $B, B / Y$ and $Y$ ions are expected to be observed in the mass spectrum, with the theory that the GlcA cleavage mechanism occurs at a lower collision energy than the IdoA mechanism.

\begin{tabular}{|c|c|c|c|c|c|c|c|}
\hline & Four ring & Six ring & Eight ring & $Y_{0}{ }^{-}$ & $Y_{2}^{-}$ & $Y_{4}^{2-}$ & $\mathrm{Y}_{6}{ }^{3-}$ \\
\hline \multicolumn{8}{|l|}{ |-I-I-I-Tag } \\
\hline I-I-I-G-Tag & & & $\checkmark$ & $\bullet$ & & & \\
\hline |-I-G-|-Tag & & $\bullet$ & & & $\bullet$ & & \\
\hline I-G-I-I-Tag & $\checkmark$ & & & & & $\bullet$ & \\
\hline G-I-I-I-tag & & & & & & & $\bullet$ \\
\hline I-I-G-G-Tag & & $\downarrow$ & 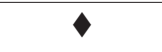 & $\checkmark$ & $\diamond$ & & \\
\hline I-G-G-I-Tag & & $\bullet$ & & & $\bullet$ & $\bullet$ & \\
\hline G-G-I-I-Tag & $\checkmark$ & & & & & $\checkmark$ & $\downarrow$ \\
\hline G-I-I-G-Tag & & $\bullet$ & $\bullet$ & $\bullet$ & & & $\bullet$ \\
\hline I-G-I-G-Tag & 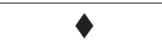 & & 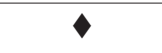 & $\downarrow$ & & $\downarrow$ & \\
\hline G-I-G-I-Tag & $\checkmark$ & 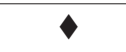 & & & $\downarrow$ & & $\checkmark$ \\
\hline G-G-G-I-Tag & $\downarrow$ & $\downarrow$ & & & 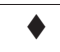 & $\downarrow$ & 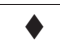 \\
\hline G-G-I-G-Tag & $\downarrow$ & $\checkmark$ & $\checkmark$ & $\downarrow$ & & $\checkmark$ & $\checkmark$ \\
\hline G-I-G-G-Tag & $\downarrow$ & $\checkmark$ & $\checkmark$ & $\checkmark$ & $\bullet$ & & $\downarrow$ \\
\hline I-G-G-G-Tag & $\diamond$ & 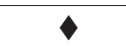 & $\checkmark$ & $\diamond$ & 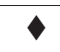 & $\bullet$ & \\
\hline G-G-G-G-Tag & $\downarrow$ & $\bullet$ & $\bullet$ & $\bullet$ & $\bullet$ & $\downarrow$ & $\bullet$ \\
\hline
\end{tabular}

an IdoA cleavage mechanism to be determined. Figure 6 and Supplementary Figure 2 show the experimental data obtained when the relative abundance of product ions for each of the isomers is compared.

A comparison of the observed data (Figure 6) with the theoretical data (Table 1) resulted in one unique sequence for each compound. Compound 3 was G-G-I-I-Tag (I-Tag, $Y_{4}^{2-}, Y_{6}^{3-}$, four ring), compound 4 was $G-I-I-G-T a g\left[Y_{0}^{-}(G-T a g), Y_{6}^{3-}\right.$, eight ring, six ring], compound 5 was I-G-I-G-Tag $\left[Y_{0}^{-}(G-T a g), Y_{4}^{2-}\right.$, eight ring, four ring), and compound 6 was G-I-G-I-Tag (I-Tag, $Y_{2}$,
$\mathrm{Y}_{6}^{3-}$, six ring, four ring). (Sequencing data for compounds 1 and 2 are discussed in the previous section.)

The approach described above should have utility for the characterization of saccharides both smaller and larger than octasaccharides, though greater challenges are expected as the degree of polymerization increases. Theoretically, it should be possible to calculate the proportion of each dissociation product. Although the proportion patterns match the theoretical patterns, not all of the compounds produced the expected ratios. This is not totally surprising given the similar ATDs
回

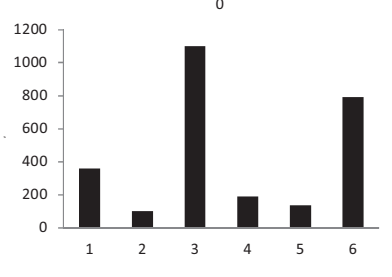

$\mathrm{B}_{8}-\mathrm{SO}_{3}$

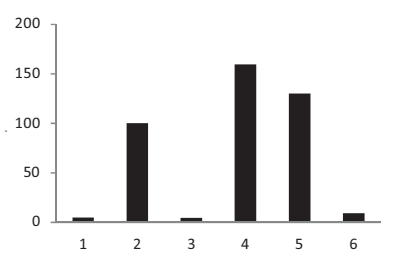

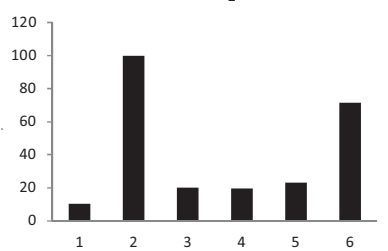

$\mathrm{B}_{6}-\mathrm{SO}_{3}, \mathrm{~B}_{8}-\mathrm{SO}_{3}$ ??ㅛㅇㅢ

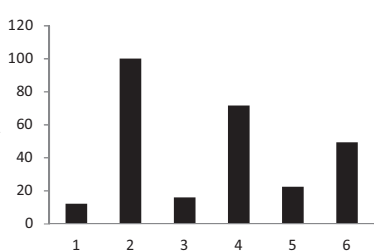

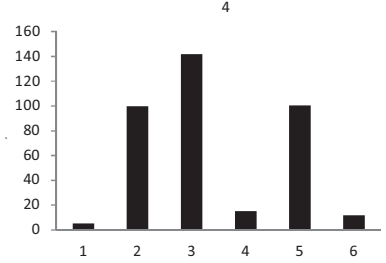

$\mathrm{B}_{4}-\mathrm{SO}_{3}, \mathrm{~B}_{6}-\mathrm{SO}_{3}$ 료령, $\mathrm{B}_{8}-\mathrm{SO}_{3}$ 리른

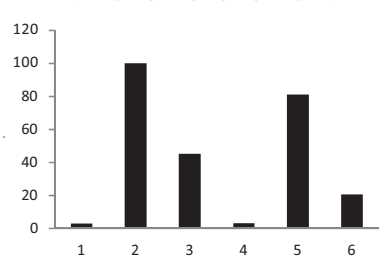

]$_{6}$

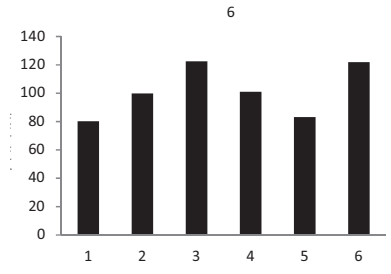

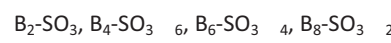

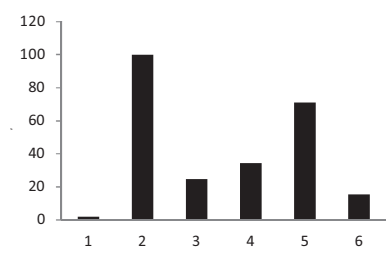

Figure 6. Glycosidic bond cleavage ion differences among compounds 1-6. Diagrammatic annotation of $\mathrm{MS}^{2}$ glycosidic bond cleavage ions (Domon and Costello nomenclature ${ }^{49}$ ) identified in compounds 1-6 when subjected to a collision energy of $22 \mathrm{~V}$. Each glycosidic cleavage ion was calculated relative to the parent ion, $\mathrm{m} / \mathrm{z} 625\left[\mathrm{M}-\mathrm{SO}_{3}-3 \mathrm{H}\right]^{3-}$, in each compound. These data were normalized against compound 2 (fully GlcA structure). 
observed for two different structures, G-G-G-G-Tag and I-G-IG-Tag. Continued studies on defined HS saccharides, including those with $\mathrm{N}$-sulfation and other $\mathrm{O}$-sulfation patterns, and improvement of our knowledge on structural conformation relative to sequences should continue in an effort to improve the accuracy of future sequencing strategies.

\section{Conclusions}

Six fully defined dp8 structures, isomeric with respect to their carboxylic acid group orientations (from axial to equatorial), were analyzed and differentiated by both ion mobility and $\mathrm{MS}^{2}$. Ion mobility suggested that a fully GlcA dp8 and a fully IdoA dp8 adopted two different conformations, with the former being more compact and the latter being more extended. Structures containing a reducing end GlcA-tag occupy a similar hydrodynamic volume, and thus may be compacting the structure. Switching the GlcA and IdoA acid positions caused the conformation to become more elongated as evidenced by the ATD. These data clearly show the importance of sequence to overall conformation, and suggest how this could affect molecular interactions with protein ligands.

Analysis of the $\mathrm{MS}^{2}$ data showed that each dp8 isomer had unique sets of product ion intensities. A comparison of the product ions created via glycosidic bond cleavages showed that the intensities were much greater when the neighboring group to the non-reducing end was a GlcA rather than an IdoA. MS ${ }^{2}$ validated and confirmed the structural differences between the various isomers, and produced characteristic and diagnostic product ions for each compound analyzed. MS $^{3}$ experiments (Supplementary Figures 3-5 and Supplementary Methods) further supported the $\mathrm{MS}^{2}$ data. Regardless of the collision energy used, the glucuronic isomer always produced a glycosidic cleavage ion that was of greater abundance than the corresponding iduronic isomer. This knowledge enabled complete GlcA or IdoA sequencing on each of the four positions located in each dp8 structure.

We are now applying ion mobility and $M^{2}$ to structures with divergent sulfation patterns, which remains a significant analytical challenge, but it is clear that MS will continue to be a crucial tool in elucidating the structure-function relationships of $\mathrm{HS}$ in biological systems.

\section{Acknowledgements}

J.A.L. and R.L.M. are grateful to Dr Youjin Seo for assistance with some of the MS experiments. This research was supported by NIH GM47356-20.

\section{Supplementary data}

Supplementary data associated with this article can be found in the online version at http://dx.doi.org/10.1255/ejms.1337.

\section{References}

1. O.N. El-Assal, A. Radulescu and G.E. Besner, "Heparinbinding EGF-like growth factor preserves mesenteric microcirculatory blood flow and protects against intestinal injury in rats subjected to hemorrhagic shock and resuscitation", Surgery 142, 234 (2007). doi: http://dx.doi. org/10.1016/i.surg.2007.04.003

2. D.V. Rocourt, V.B. Mehta and G.E. Besner, "Heparinbinding EGF-like growth factor decreases inflammatory cytokine expression after intestinal ischemia/reperfusion injury", J. Surg. Res. 139, 269 (2007). doi: http:// dx.doi.org/10.1016/j.jss.2006.10.047

3. U. Lindahl, L. Thunberg, G. Backstrom and J. Riesenfeld, "The antithrombin-binding sequence of heparin",

Biochem. Soc. Trans. 9, 499 (1981). doi: http://dx.doi. org/10.1042/bst0090499

4. R. Copeland, A. Balasubramaniam, V. Tiwari, F. Zhang, A. Bridges, R. J. Linhardt, D. Shukla and J. Liu, "Using a 3-0-sulfated heparin octasaccharide to inhibit the entry of Herpes simplex virus type 1", Biochemistry 47, 5774 (2008). doi: http://dx.doi.org/10.1021/bi800205t

5. A. Ori, M.C. Wilkinson and D.G. Fernig, "The heparanome and regulation of cell function: structures, functions and challenges", Front. Biosci. 13, 4309 (2007). doi: http://dx.doi.org/10.2741/3007

6. M. Klagsbrun, "Mediators of angiogenesis: the biological significance of basic fibroblast growth factor (BFGF)heparin and heparan sulfate interactions", Semin. Cancer Biol. 1992, 81 (1992).

7. T. Sasaki, H. Larsson, J. Kreuger, M. Salmivirta, L. Claesson-Welsh, U. Lindahl, E. Hohenester and R. Timpl, "Structural basis and potential role of heparin/ heparan sulfate binding to the angiogenesis inhibitor endostatin", EMBO J. 18, 6240 (1999). doi: http://dx.doi. org/10.1093/emboj/18.22.6240

8. Z. Hu, C. Wang, Y. Xiao, N. Sheng, Y. Chen, Y. Xu, L. Zhang, W. Mo, N. Jing and G. Hu, "NDST1-dependent heparan sulfate regulates BMP signaling and internalization in lung development", J. Cell Sci. 122, 1145 (2009). doi: http://dx.doi.org/ 10.1242/jcs.034736

9. H. Zhou, S. Roy, E. Cochran, R. Zouaoui, C.L. Chu, J. Duffner, G. Zhao, S. Smith, Z. Galcheva-Gargova and J. Karlgren, "M402, a novel heparan sulfate mimetic, targets multiple pathways implicated in tumor progression and metastasis", PLoS ONE 6, e21106 (2011). doi: http://dx.doi.org/10.1371/journal.pone.0021106

10. L. Jin, J.P. Abrahams, R. Skinner, M. Petitou, R.N. Pike and R.W. Carrell, "The anticoagulant activation of antithrombin by heparin", Proc. Natl. Acad. Sci. 94, 14683 (1997). doi: http://dx.doi.org/10.1073/ pnas.94.26.14683

11. M. Petitou, B. Casu and U. Lindahl, “1976-1983, a critical period in the history of heparin: the discovery of the antithrombin binding site", Biochimie 85, 83 (2003). doi: http://dx.doi.org/10.1016/S0300-9084(03)00078-6 
12. K.J. Bame, I. Venkatesan, H.D. Stelling and S. Tumova, "The spacing of S-domains on HS glycosaminoglycans determines whether the chain is a substrate for intracellular heparanases", Glycobiology 10, 715 (2000). doi: http://dx.doi.org/10.1093/glycob/10.7.715

13. M. Lyon and J.T. Gallagher, "Bio-specific sequences and domains in heparan sulphate and the regulation of cell growth and adhesion", Matrix Biol. 17, 485 (1998). doi: http://dx.doi.org/10.1016/S0945-053X(98)90096-8

14. D.H. Atha, J.C. Lormeau, M. Petitou, R.D. Rosenberg and J. Choay, "Contribution of monosaccharide residues in heparin binding to antithrombin III", Biochemistry 24, 6723 (1985). doi: http://dx.doi.org/10.1021/bi00344a063

15. U. Lindahl, G. Bäckström and L. Thunberg, "The antithrombin-binding sequence in heparin. Identification of an essential 6-0-sulfate group", J. Biol. Chem. 258, 9826 (1983).

16. U. Lindahl, G. Bäckström, L. Thunberg and I.G. Leder, "Evidence for a 3-0-sulfated D-glucosamine residue in the antithrombin-binding sequence of heparin", Proc. Natl. Acad. Sci. 77, 6551 (1980). doi: http://dx.doi. org/10.1073/pnas.77.11.6551

17. M. Petitou, P. Duchaussoy, I. Lederman, J. Choay and P. Sinaÿ, "Binding of heparin to antithrombin III: a chemical proof of the critical role played by a 3-sulfated 2-amino-2-deoxy-D-glucose residue", Carbohydr. Res. 179, 163 (1988). doi: http://dx.doi. org/10.1016/0008-6215(88)84116-8

18. J. Riesenfeld, L. Thunberg, M. Höök and U. Lindahl, "The antithrombin-binding sequence of heparin. Location of essential N-sulfate groups", J. Biol. Chem. 256, 2389 (1981).

19. C.L. Merry, M. Lyon, J.A. Deakin, J.J. Hopwood and J.T. Gallagher, "Highly sensitive sequencing of the sulfated domains of heparan sulfate", J. Biol. Chem. 274, 18455 (1999). doi: http://dx.doi.org/10.1074/jbc.274.26.18455

20. O.M. Saad, H. Ebel, K. Uchimura, S.D. Rosen, C.R. Bertozzi and J.A. Leary, "Compositional profiling of heparin/heparan sulfate using mass spectrometry: assay for specificity of a novel extracellular human endosulfatase", Glycobiology 15, 818 (2005). doi: http://dx.doi. org/10.1093/glycob/cwi064

21. O.M. Saad and J.A. Leary, "Compositional analysis and quantification of heparin and heparan sulfate by electrospray ionization ion trap mass spectrometry", Anal. Chem. 75, 2985 (2003). doi: http://dx.doi.org/10.1021/ ac0340455

22. O.M. Saad and J.A. Leary, "Delineating mechanisms of dissociation for isomeric heparin disaccharides using isotope labeling and ion trap tandem mass spectrometry", J. Am. Soc. Mass Spectrom. 15, 1274 (2004). doi: http://dx.doi.org/10.1016/j.jasms.2004.05.008

23. O.M. Saad and J.A. Leary, "Heparin sequencing using enzymatic digestion and ESI-MSn with host: a heparin/ HS oligosaccharide sequencing tool", Anal. Chem. 77, 5902 (2005). doi: http://dx.doi.org/10.1021/ac050793d
24. M.R. Schenauer, J.K. Meissen, Y. Seo, J.B. Ames and J.A. Leary, "Heparan sulfate separation, sequencing, and isomeric differentiation: ion mobility spectrometry reveals specific iduronic and glucuronic acid-containing hexasaccharides", Anal. Chem. 81, 10179 (2009). doi: http://dx.doi.org/10.1021/ac902186h

25. Y. Seo, A. Andaya and J.A. Leary, "Preparation, separation, and conformational analysis of differentially sulfated heparin octasaccharide isomers using ion mobility mass spectrometry", Anal. Chem. 84, 2416 (2012). doi: http://dx.doi.org/10.1021/ac203190k

26. R. Vives, D. Pye, M. Salmivirta, J. Hopwood, U. Lindahl and J. Gallagher, "Sequence analysis of heparan sulphate and heparin oligosaccharides", Biochem. J. 339, 767 (1999). doi: http://dx.doi.org/10.1042/0264$\underline{6021: 3390767}$

27. J.E. Turnbull, J.J. Hopwood and J.T. Gallagher, "A strategy for rapid sequencing of heparan sulfate and heparin saccharides", Proc. Natl. Acad. Sci. 96, 2698 (1999). doi: http://dx.doi.org/10.1073/pnas.96.6.2698

28. G. Venkataraman, Z. Shriver, R. Raman and R. Sasisekharan, "Sequencing complex polysaccharides", Science 286, 537 (1999). doi: http://dx.doi.org/10.1126/science.286.5439.537

29. M. Hricovíni and G. Torri, “Dynamics in aqueous solutions of the pentasaccharide corresponding to the binding site of heparin for antithrombin III studied by NMR relaxation measurements", Carbohydr. Res. 268, 159 (1995). doi: http://dx.doi. org/10.1016/0008-6215(94)00334-C

30. F.E. Leach III, S. Arungundram, K. Al-Mafraji, A. Venot, G.-J. Boons and I.J. Amster, "Electron detachment dissociation of synthetic heparan sulfate glycosaminoglycan tetrasaccharides varying in degree of sulfation and hexuronic acid stereochemistry", Int. J. Mass Spectrom. 330, 152 (2012). doi: http://dx.doi.org/10.1016/j. ijms.2012.07.002

31. B. Mulloy and M.J. Forster, "Conformation and dynamics of heparin and heparan sulfate", Glycobiology 10, 1147 (2000). doi: http://dx.doi.org/10.1093/glycob/10.11.1147

32. H.B. Oh, F.E. Leach III, S. Arungundram, K. Al-Mafraji, A. Venot, G.-J. Boons and I.J. Amster, "Multivariate analysis of electron detachment dissociation and infrared multiphoton dissociation mass spectra of heparan sulfate tetrasaccharides differing only in hexuronic acid stereochemistry", J. Am. Soc. Mass Spectrom. 22, 582 (2011). doi: http://dx.doi.org/10.1007/s13361-010-0047-y

33. J.J. Wolff, L. Chi, R. J. Linhardt and I.J. Amster, "Distinguishing glucuronic from iduronic acid in glycosaminoglycan tetrasaccharides by using electron detachment dissociation", Anal. Chem. 79, 2015 (2007). doi: http://dx.doi.org/10.1021/ac061636x

34. F.E. Leach III, J.J. Wolff, Z. Xiao, M. Ly, T.N. Laremore, S. Arungundram, K. Al-Mafraji, A. Venot, G.-J. Boons and R.J. Linhardt, "Negative electron transfer dissociation Fourier transform mass spectrometry 
of glycosaminoglycan carbohydrates", Eur. J. Mass Spectrom. 17, 167 (2011). doi: http://dx.doi.org/10.1255/ ejms. 1120

35. J.J. Wolff, F.E. Leach III, T.N. Laremore, D.A. Kaplan, M.L. Easterling, R.J. Linhardt and I.J. Amster, "Negative electron transfer dissociation of glycosaminoglycans", Anal. Chem. 82, 3460 (2010). doi: http://dx.doi. org/10.1021/ac100554a

36. J.J. Wolff, I.J. Amster, L. Chi and R. J. Linhardt, "Electron detachment dissociation of glycosaminoglycan tetrasaccharides", J. Am. Soc. Mass Spectrom. 18, 234 (2007). doi: http://dx.doi.org/10.1016/j.jasms.2006.09.020

37. J.J. Wolff, T.N. Laremore, A.M. Busch, R. J. Linhardt and I.J. Amster, "Electron detachment dissociation of dermatan sulfate oligosaccharides", J. Am. Soc. Mass Spectrom. 19, 294 (2008). doi: http://dx.doi.org/10.1016/j. jasms.2007.10.007

38. J.J. Wolff, T.N. Laremore, H. Aslam, R.J. Linhardt and I.J. Amster, "Electron-induced dissociation of glycosaminoglycan tetrasaccharides", J. Am. Soc. Mass Spectrom. 19, 1449 (2008). doi: http://dx.doi.org/10.1016/j. jasms.2008.06.024

39. E. Atkins and T. Laurent, "X-ray diffraction patterns from chondroitin 4-sulphate, dermatan sulphate and heparan sulphate", Biochem. J. 133, 605 (1973).

40. D.R. Ferro, A. Provasoli, M. Ragazzi, B. Casu, G. Torri, V. Bossennec, B. Perly, P. Sinä̈, M. Petitou and J. Choay, "Conformer populations of L-iduronic acid residues in glycosaminoglycan sequences", Carbohydr. Res. 195, 157 (1990). doi: http://dx.doi. org/10.1016/0008-6215(90)84164-P

41. P.N. Sanderson, T.N. Huckerby and I.A. Nieduszynski, "Conformational equilibria of alpha-L-iduronate residues in disaccharides derived from heparin", Biochem. J. 243, 175 (1987).

42. E.A. Yates, F. Santini, M. Guerrini, A. Naggi, G. Torri and $\mathrm{B}$. Casu, ${ }^{11} \mathrm{H}$ and ${ }^{13} \mathrm{C}$ NMR spectral assignments of the major sequences of twelve systematically modified heparin derivatives", Carbohydr. Res. 294, 15 (1996). doi: http://dx.doi.org/10.1016/S0008-6215(96)90611-4
43. Y. Seo, A. Andaya, C. Bleiholder and J.A. Leary, "Differentiation of CC vs CXC chemokine dimers with GAG octasaccharide binding partners: an ion mobility mass spectrometry approach", J. Am. Chem. Soc. 135, 4325 (2013). doi: http://dx.doi.org/10.1021/ja310915m

44. Y. Seo, M.R. Schenauer and J.A. Leary, "Biologically relevant metal-cation binding induces conformational changes in heparin oligosaccharides as measured by ion mobility mass spectrometry", Int. J. Mass Spectrom. 303, 191 (2011). doi: http://dx.doi.org/10.1016/j. ijms.2011.02.003

45. J. Ujma, M. De Cecco, O. Chepelin, H. Levene, C. Moffat, S.J. Pike, P.J. Lusby and P.E. Barran, "Shapes of supramolecular cages by ion mobility mass spectrometry", Chem. Commun. 48, 4423 (2012). doi: http://dx.doi. org/10.1039/C2CC30778B

46. L. Jin, P.E. Barran, J.A. Deakin, M. Lyon and D. Uhrín, "Conformation of glycosaminoglycans by ion mobility mass spectrometry and molecular modelling", Phys. Chem. Chem. Phys. 7, 3464 (2005). doi: http://dx.doi. org/10.1039/B508644B

47. E. Jurneczko, J. Kalapothakis, I.D. Campuzano, M. Morris and P.E. Barran, "Effects of drift gas on collision cross sections of a protein standard in linear drift tube and traveling wave ion mobility mass spectrometry", Anal. Chem. 84, 8524 (2012). doi: http://dx.doi. org/10.1021/ac301260d

48. R. Schwörer, O.V. Zubkova, J.E. Turnbull and P.C. Tyler, "Synthesis of a targeted library of heparan sulfate hexa $\beta$ to dodecasaccharides as inhibitors of $\beta$-secretase: potential therapeutics for Alzheimer's disease", Chem. Eur. J. 19, 6817 (2013). doi: http://dx.doi.org/10.1002/ chem.201204519

49. B. Domon and C.E. Costello, "Structure elucidation of glycosphingolipids and gangliosides using high-performance tandem mass spectrometry", Biochemistry 27, 1534 (1988). doi: http://dx.doi.org/10.1021/bi00405a021 